\title{
RESISTENCIA DE M. TUBERCULOSIS A LAS DROGAS
}

\author{
LUIS CARLOS OROZCO, * GUILLERMO APARICIO, ** OTILIA QUINTERO DE RAMOS, ${ }^{* *}$ \\ ESNEDA GIRALDO DE BLANCO, ${ }^{* * *}$ ISABEL ULLOA DE MORENO, ${ }^{* * *}$ CLARA INES LEON FRANCO, ${ }^{* *}$
}

\begin{abstract}
Se estudian y analizan retrospectivamente los resultados de las pruebas de sensibilidad de $M$. tuberculosis a diferentes drogas antituberculosas, realizadas en el Instituto Nacional de Salud, durante un período de 11 años (1970-1980).
\end{abstract}

Fue posible demostrar:

1. Un notorio aumento de la resistencia a la INH y Tb1, que se estabiliza desde 1975 hasta la fecha.

2. La resistencia a la RMP evidencia un preocupante incremento.

3. Las drogas restantes SM, PAS, TRE y CS presentan una disminución considerable de la resistencia.

\section{INTRODUCCION}

Se define como resistencia de $M$. tuberculosis a una droga: "a la disminución de grado suficiente en la sensibilidad, que nos da una razonable certeza que tal cepa es diferente a un grupo de cepas de tipo humano que nunca han estado en contacto con la droga"'(1).

La resistencia de $M$. tuberculosis a una y aún a dos drogas antituberculosas, no parece influir en forma significativa en la rata de curación de los pacientes regulares,

\footnotetext{
* M.D. Jefe Encargado Grupo de Micobacterias, Instituto Nacional de Salud.

** M.D. Jefe Titular Grupo de Micobacterias, Instituto Nacional de Salud.

*** Bacteriólogas Grupo de Micobacterias, Instituto Nacional de Salud.
}

con relación a la ingesta de los tratamientos bi o triconjugados (2). Canetti, et al. propusieron en 1969 que el estudio periódico de las resistencias de $M$. tuberculosis, permitiría evaluar los programas de lucha antituberculosa, al demostrarse que el porcentaje de resistencia a los antibióticos o quimioterápicos "se estabilizaba o aún tendía a la disminución, cuando los programas de control de la tuberculosis mejoraban"'(3).

Lo anterior sólo era posible realizarlo a través de estudios de prevalencia de resistencia inicial y primaria, investigaciones no solamente costosas sino difíciles de realizar, en un país donde la disponibilidad del cultivo no es alta en todos los niveles.

El estudio de la resistencia de $M$. tuberculosis a las drogas se inició en Colombia en 1964, con un método semicuantitativo que fue cambiado en 1970 por el método de las 
proporciones múltiples, que no solamente es más reproducible sino que además permite un análisis cuantitativo (3).

\section{MATERIALES Y METODOS}

El presente estudio se realizó con base en los datos obtenidos por el Laboratorio de Tuberculosis del Grupo de Micobacterias del Instituto Nacional de Salud entre los años 1970 y 1980 , sobre las pruebas de resistencia efectuadas por el método de las proporciones múltiples, a los cultivos de $M$. tuberculosis, remitidos por los laboratorios periféricos del Programa de Tuberculosis del Ministerio de Salud y algunas Universidades, con la sospecha clínica de resistencia a las drogas antituberculosas.

Se clasificaron los datos en forma anual, para obtener el número total de cepas estudiadas, el número total de cepas sensibles, las cuales al descontarse del total de cepas estudiadas, nos dieron el total de cepas resistentes a por lo menos una droga; con este número como denominador y el de cepas resistentes a cada una de las drogas como numerador, se obtuvo el porcentaje de la resistencia a cada una de las drogas de primera línea: Isoniacida (INH), Estreptomicina (SM), Thioacetazona (Tb1), Acido Paraminosalicílico (PAS), y de segunda línea: Etambutol (EMB), Trecator (TRE), Cicloserina (CS), Rifampicina (RMP).

Posteriormente se realizó una prueba de significación estadística $\mathrm{X}^{2}$ (chi cuadrado), para investigar si las diferencias observadas con cada una de las drogas, a lo largo de los once años, eran significativas o no; esto se graficó en forma tal que se pudiera comparar cada año con los demás. La prueba de significación estadística a la RMP sólo fue analizada comparando el año de 1972 con el de 1980. Se utilizó en todos los casos $\mathrm{p} \leq$ 0.05 .

\section{RESULTADOS}

En la tabla No. 1 se presenta el total de cepas estudiadas en forma anual, y la distribución porcentual de cepas resistentes y sensibles. Se observa un incremento progresivo del número total de pruebas realizadas hasta 1975 , años después del cual, vuelve a disminuír. Se observa una gran variabilidad, sin tendencia definida, a lo largo de los años, en el porcentaje de resistencia, que oscila entre $83 \%$ en 1970 y $48 \%$ en 1975 . Los porcentajes de resistencia y sensibilidad nos permitieron realizar una gráfica de certeza, ante la sospecha clínica de resistencia, (figura No. 1). Las tablas Nos. 2 y 3 muestran los porcentajes anuales de resistencia a las diferentes drogas, fenómeno que se aprecia mejor en las figuras 2,3 y 4.

TABLA NE I. TOTAL PRUEBAS REALIZADAS. PORCENTAJES DE RESISTENCIA Y SENSIBILIDAD

\begin{tabular}{|c|c|c|c|c|c|c|c|c|c|c|c|}
\hline & 70 & 71 & 72 & 73 & 74 & 75 & 76 & 77 & 78 & 79 & 80 \\
\hline $\begin{array}{l}\text { N2 TOTAL } \\
\text { PRUEBAS }\end{array}$ & 159 & 411 & 894 & 940 & 1528 & 1350 & 1165 & 674 & 680 & 809 & 870 \\
\hline$\% \mathrm{R}$ & 83.0 & 58.4 & 54.9 & 62.3 & 53.7 & 47.8 & 54.6 & 72.0 & 67.5 & 65.8 & 63.1 \\
\hline$\% \mathrm{~S}$ & 17.0 & 41.6 & 45.1 & 37.7 & 46.3 & 52.2 & 45.4 & 28.0 & 32.5 & 34.2 & 36.9 \\
\hline
\end{tabular}

$\%$

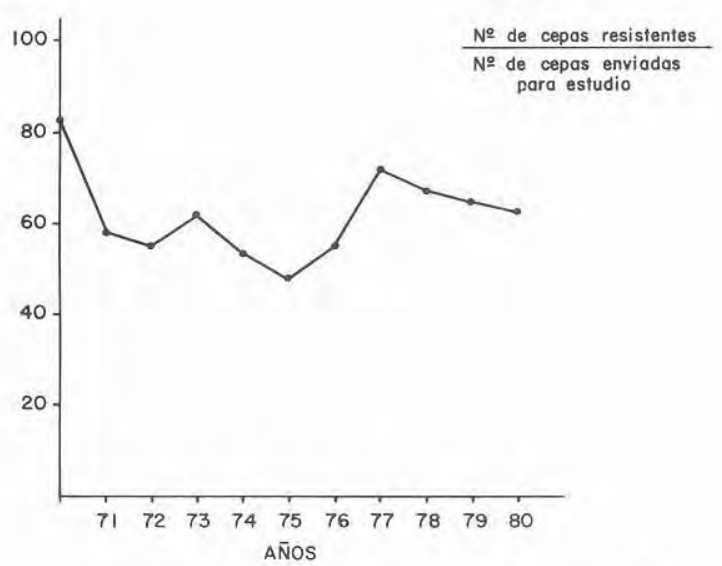

Figura № I. CERTEZA CLINICA DE RESISTENCIA

TABLA NE 2. RESISTENCIA A DROGAS DE PRIMERA LINEA.

\begin{tabular}{|l|c|c|c|c|c|c|c|c|c|c|c|}
\hline PROGA & 70 & 71 & 72 & 73 & 74 & 75 & 76 & 77 & 78 & 79 & 80 \\
\hline ANO & 24.2 & 5.0 & 4.5 & 9.4 & 12.8 & 11.5 & 7.1 & 5.4 & 5.0 & 8.6 & 9.5 \\
\hline TR & 38.6 & 23.8 & 20.8 & 18.6 & 26.1 & 21.9 & 8.0 & 4.3 & 5.2 & 3.4 & 5.3 \\
\hline CS & 14.4 & 6.3 & 2.2 & 0.8 & 1.7 & 1.4 & 0.6 & 2.7 & 1.5 & 2.6 & 0.9 \\
\hline RMP & - & - & 1.0 & - & - & - & 0 & 1.0 & 0.7 & 3.0 & 5.3 \\
\hline
\end{tabular}




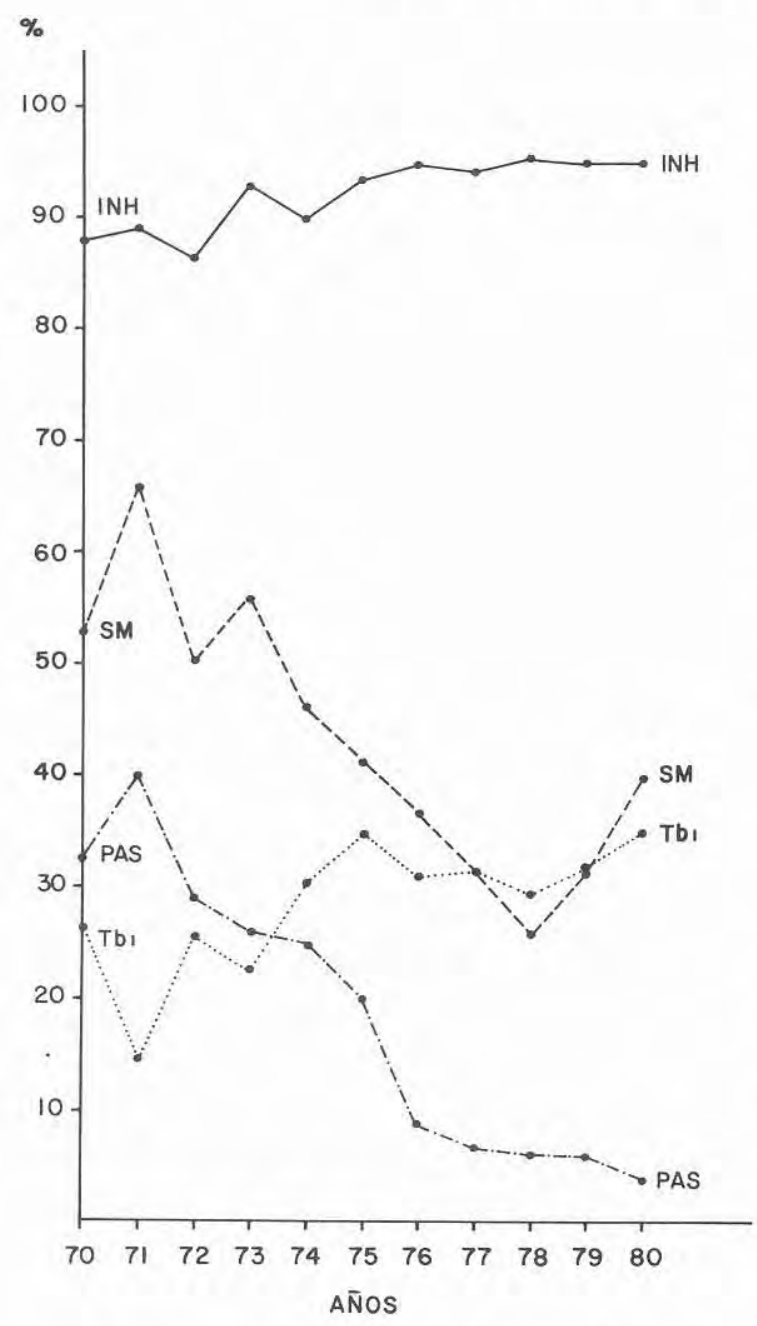

Figura № 2. RESISTENCIA A DROGAS DE PRI MERA LINEA.

TABLA N2 3. RESISTENCIA A DROGAS dE SEGUNDA LINEA.

\begin{tabular}{|l|c|c|c|c|c|c|c|c|c|c|c|}
\hline PROGA & 70 & 71 & 72 & 73 & 74 & 75 & 76 & 77 & 78 & 79 & 80 \\
\hline INH & 88.6 & 89.2 & 87.6 & 95.4 & 90.1 & 93.2 & 94.7 & 94.4 & 95.6 & 94.7 & 95.1 \\
\hline TbI & 26.5 & 14.6 & 25.7 & 22.5 & 30.6 & 34.7 & 31.3 & 33.0 & 29.4 & 33.3 & 35.3 \\
\hline SM & 53.0 & 66.3 & 50.3 & 56.7 & 46.4 & 42.3 & 37.7 & 32.8 & 26.8 & 32.5 & 40.1 \\
\hline PAS & 32.6 & 40.0 & 29.5 & 25.9 & 25.5 & 20.0 & 8.6 & 6.8 & 6.5 & 6.6 & 3.6 \\
\hline
\end{tabular}

Al comparar los porcentajes de resistencia a las drogas en el año 1970 con los de 1980 , se encuentra que solamente aumentaron los de la INH, Tb1 y la RMP, mientras que los de las demás drogas disminuyeron; la RMP aumentó del 1\% en 1972 a $5 \%$ en 1980 (figura No. 5).

Al analizar los cambios anuales en la resistencia, buscando la significación estadística entre las diferencias, para $\mathrm{p} \leqslant 0.05$, se encuentra lo siguiente:

1. INH: aumento significativo durante los últimos años, al compararla con los primeros, con estabilización a partir de 1975.

2. Tb1: el fenómeno es similar al anterior pero con estabilización a partir de 1974 .

3. SM: la disminución porcentual, al comparar los primeros cinco años con los últimos seis, es altamente significativa.

4. PAS y TRE: se comportan como en la SM.

5. CS: disminuye en forma significativa hasta 1972 y se estabiliza a partir de ese año.

6. EMB: el comportamiento es muy irregular.

7. RMP: muestra aumento significativo al comparar las cifras de 1972 con las de 1980.

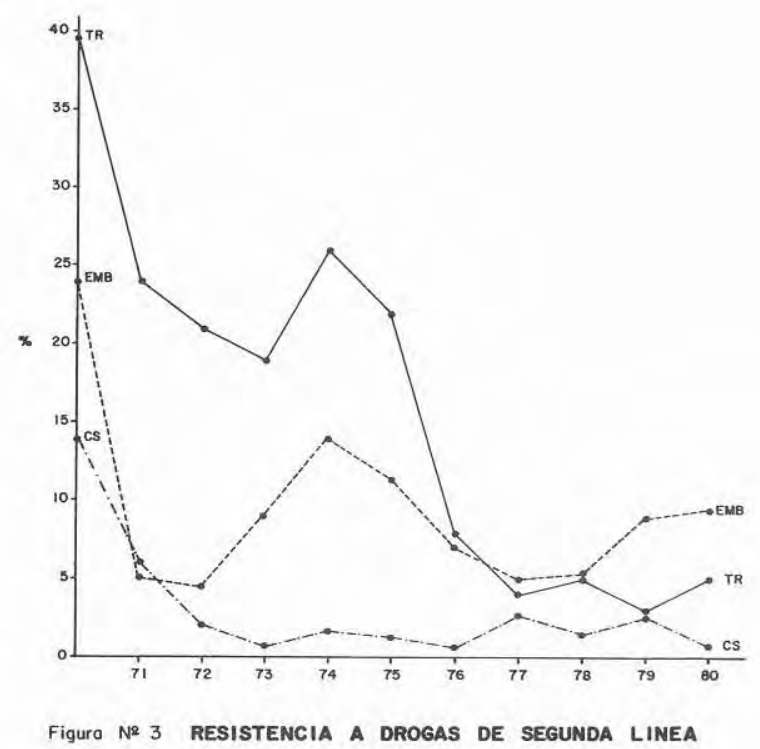




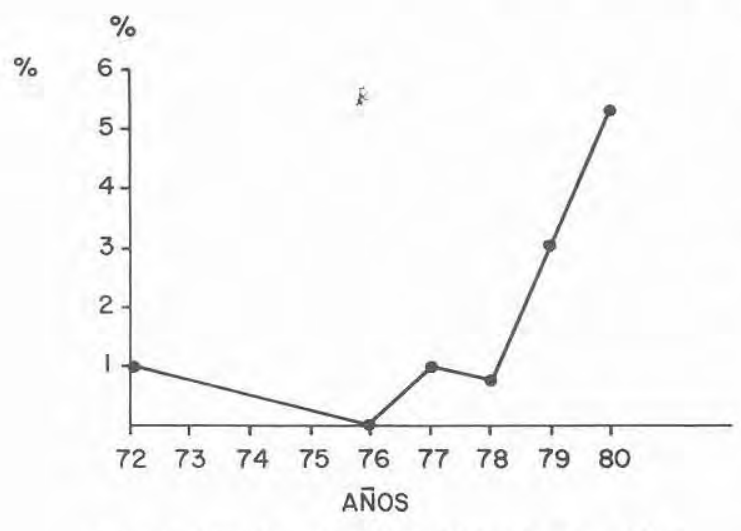

Figura № 4. RESISTENCIA A RMP

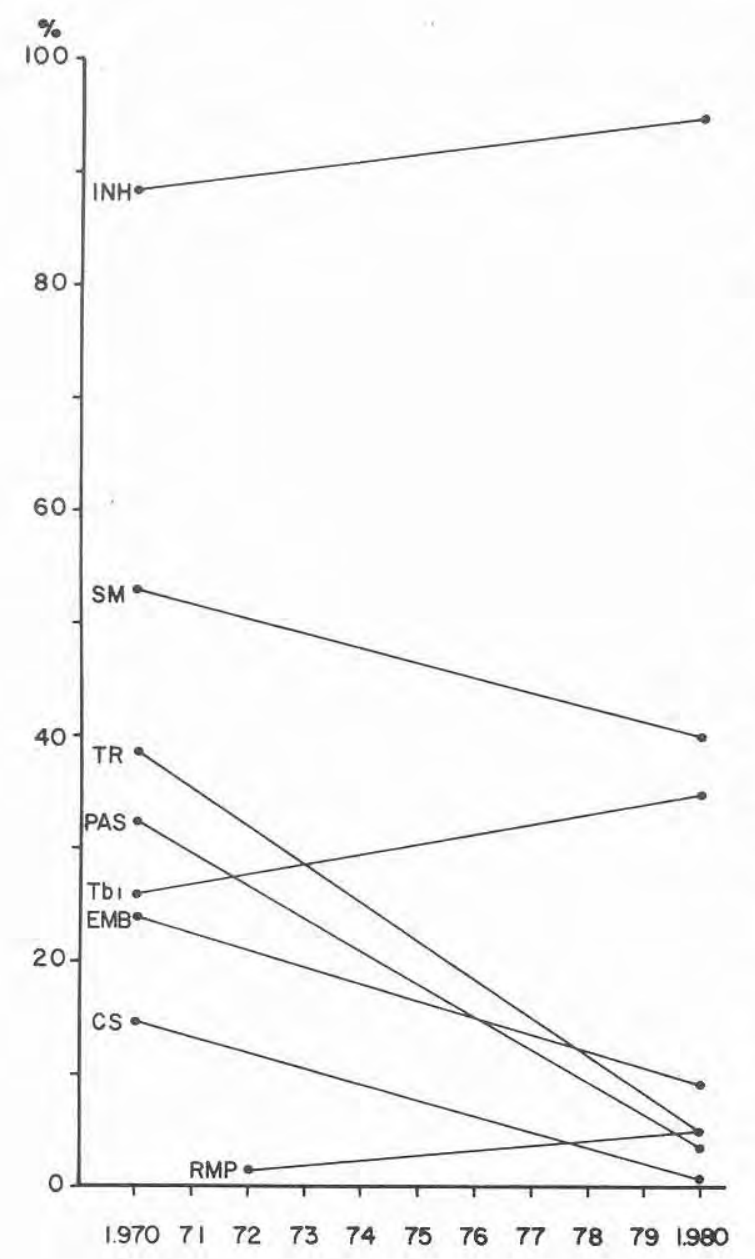

Figura № 5. COMPARACION DE RESISTEN CIAS ENTRE 1.970 Y 1.980 .

\section{DISCUSION}

Las pruebas de resistencia en el país, son realizadas por solicitud de los laboratorios periféricos del Programa Antituberculoso, con base en la sospecha clínica de falla terapéutica por resistencia; por esta razón, el actual estudio no permite calcular los porcentajes de resistencias primaria*, inicial ${ }^{* *}$, ni secundaria ${ }^{* * *}$.

Al observar la gráfica de certeza clínica de resistencia, vemos como en los años 1971, $72,74,75$ y 76 , la falla terapéutica en alrededor de la mitad de los casos, no se debió a la presunción clínica de resistencia. Esto es debido a que la falla terapéutica en la mayoría de los casos se debe a prescripción de regímenes inadecuados, irregularidad en la ingesta de las drogas, suspensión prematura de las drogas o toxicidad de las mismas (4).

Creemos que el fenómeno de resistencia observado con el PAS, el TRE y la CS no es más que el reflejo del abandono progresivo, a partir de 1970, del uso de estas drogas en los esquemas terapéuticos antituberculosos; además este descenso progresivo permite aseverar que los resultados observados con las otras drogas son bastante confiables.

El aumento de la resistencia a la INH y Tb1 y su posterior estabilización se debe posiblemente a los siguientes fenómenos:

1. Al establecerse como criterio diagnóstico, la positividad baciloscópica de los sintomáticos respiratorios, se amplió la cobertura del diagnóstico; se incrementó así el número de pacientes en tratamiento, lo cual produce en cualquier programa, un aumento en los casos de irregularidad en la ingesta; ésto

\footnotetext{
* Resistencia primaria: la que se presenta en un enfermo que fue infectado con cepa resistente.

* Resistencia secundaria: la que se desarrolla en un individuo a lo largo de tratamientos irregulares.

*** Resistencia inicial: la de un paciente nuevo que no da una historiaconfiable sobre quimioterapia anterior. Incluiría a la primaria verdadera y a resistencia secundaria no descubierta.
} 
explicaría el aumento observado de la resistencia.

2. Al mejorar la calidad operativa en el control de los pacientes, la irregularidad de la ingesta, que fue de $55 \%$ en 1966 , descendió hasta $23 \%$ en 1974 y 1975 ; esta disminución explica la estabilización de dicha resistencia (5).

La disminución de la resistencia a la SM, es probablemente debido al mismo fenómeno anterior, es decir a un aumento en la regularidad del uso de las drogas y a la formulación de esquemas terapéuticos más adecuados.

Con relación a la irregularidad de la resistencia al EMB, una probable explicación sería la utilización, también irregular del mismo, en los tratamientos prescritos.

El aumento significativo de la resistencia a la RMP, casi vertical en los últimos tres años, aunque fue solo de $5 \%$ en 1980 , puede llevar, si esta tendencia continúa a perder una droga de alta efectividad en el tratamiento de la tuberculosis. Para evitar lo anterior sería recomendable que la RMP se restringiera en forma exclusiva a los tratamientos de la tuberculosis, los cuales cuando incluyen esta droga deben ser controlados, es decir, que el paciente ingiere la droga en presencia del personal de salud.

\section{CONCLUSIONES}

1. Aunque la resistencia es una causa de falla terapéutica en tuberculosis, no constituye la más frecuente.

2. Las drogas de segunda línea, excepción hecha del etambutol, demuestran una disminución significativa en la resistencia, lo cual les vuelve a dar un valor como drogas de elección en los re-tratamientos.

3. El uso de la RMP debe ser hecho en forma racional y bajo vigilancia estricta para evitar mayor aumento en la resistencia; idealmente solo debe usarse para la terapia antituberculosa.

4. Se debe efectuar un estudio prospectivo continuado sobre resistencia primaria para valorar las variables del fenómeno.

\section{SUMMARY}

The results of the sensitivity tests of $M$. tuberculosis to anti-tuberculous drugs, performed over a period of 11 years (1970 1980) at the Instituto Nacional de Salud of Colombia are studied and analyzed.

It was found:

1. A notorious increase in resistance to INH and Tb1, wich remains stationary since 1975 up to the present.

2. Resistance to RMP shows also a worrysome increase.

3. For the remaining drugs SM, PAS, TRE and CS there is an increase of its sensitivity.

\section{B IB LIOGRAFIA}

1. Mitchinson DA. Bulletin Int. Union against Tuberculosis $1962 ; 32: 81$ (citado en 3 ).

2. Toman K. Tuberculosis case finding and chemotherapy. WHO 1979; p 167-172.

3. Canetti G, Wallace F, Khomenko A, Mahler HT, Menon NK, Mitchinson DA, Rist N, Smelev NA. Advances intechniques of testing Mycobacterial drug sensitivity and the use of sensitivity test in tuberculosis control programmes. Bull WHO 1969; 41: 21.

4. Fox W. General considerations in the choice and management of regimens of chemotherapy for pulmonary tuberculosis. Bulletin Int. Union against Tuberculosis 1972; 47: 49.

5. Guerrero-Sandino E. Programa de Control de Tuberculosis en Colombia. Bol. Of. Sanit. Panam. 1979; $86: 398$. 